\title{
Momentum-dependent two-loop QCD corrections to the neutral Higgs-boson masses in the MSSM
}

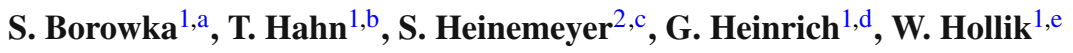 \\ ${ }^{1}$ Max-Planck-Institut für Physik (Werner-Heisenberg-Institut), Föhringer Ring 6, 80805 Munich, Germany \\ ${ }^{2}$ Instituto de Física de Cantabria (CSIC-UC), Santander, Spain
}

Received: 16 June 2014 / Accepted: 19 July 2014 / Published online: 12 August 2014

(C) The Author(s) 2014. This article is published with open access at Springerlink.com

\begin{abstract}
Results are presented for the momentum-dependent two-loop contributions of $\mathcal{O}\left(\alpha_{t} \alpha_{s}\right)$ to the masses and mixing effects in the Higgs sector of the MSSM. They are obtained in the Feynman-diagrammatic approach using a mixed on-shell/ $\overline{\mathrm{DR}}$ renormalization that can directly be matched onto the higher-order corrections included in the code FeynHiggs. The new two-loop diagrams are evaluated with the program SecDec. The combination of the new momentum-dependent two-loop contribution with the existing one- and two-loop corrections in the on-shell//DR scheme leads to an improved prediction of the light MSSM Higgs boson mass and a correspondingly reduced theoretical uncertainty. We find that the corresponding shifts in the lightest Higgs-boson mass $M_{h}$ are below $1 \mathrm{GeV}$ in all scenarios considered, but they can extend up to the level of the current experimental uncertainty. The results are included in the code FeynHiggs.
\end{abstract}

\section{Introduction}

The ATLAS and CMS experiments at CERN have recently discovered a new boson with a mass around $125.6 \mathrm{GeV}$ [1,2]. Within the present experimental uncertainties this new boson behaves like the Higgs boson of the Standard Model (SM) [36]. However, the newly discovered particle can also be interpreted as the Higgs boson of extended models. The Higgs sector of the Minimal Supersymmetric Standard Model (MSSM) [7-9] with two scalar doublets accommodates five physi-

\footnotetext{
a e-mail: sborowka@mpp.mpg.de

b e-mail: hahn@mpp.mpg.de

c e-mail: Sven.Heinemeyer@cern.ch

de-mail: gudrun@mpp.mpg.de

e e-mail: hollik@mpp.mpg.de
}

cal Higgs bosons. In lowest order these are the light and heavy $\mathcal{C P}$-even $h$ and $H$, the $\mathcal{C P}$-odd $A$, and the charged Higgs bosons $H^{ \pm}$. The measured mass value, having already reached the level of a precision observable with an experimental accuracy of about $500 \mathrm{MeV}$, plays an important role in this context. In the MSSM the mass of the light $\mathcal{C P}$-even Higgs boson, $M_{h}$, can directly be predicted from the other parameters of the model. The accuracy of this prediction should at least match the one of the experimental result.

The Higgs sector of the MSSM can be expressed at lowest order in terms of the gauge couplings, the mass of the $\mathcal{C P}$-odd Higgs boson, $M_{A}$, and $\tan \beta \equiv v_{2} / v_{1}$, the ratio of the two vacuum expectation values. All other masses and mixing angles can therefore be predicted. Higher-order contributions can give large corrections to the tree-level relations [10-12]. An upper bound for the mass of the lightest MSSM Higgs boson of $M_{h} \lesssim 135 \mathrm{GeV}$ was obtained [13], and the remaining theoretical uncertainty in the calculation of $M_{h}$, from unknown higher-order corrections, was estimated to be up to $3 \mathrm{GeV}$, depending on the parameter region. Recent improvements have lead to a somewhat smaller estimate of up to $\sim 2 \mathrm{GeV}[14,15]$ (see below).

Experimental searches for the neutral MSSM Higgs bosons have been performed at LEP $[16,17]$, placing important restrictions on the parameter space. At Run II of the Tevatron the search was continued but is now superseded by the LHC Higgs searches. Besides the discovery of a SM Higgs-like boson the LHC searches place stringent bounds, in particular in the regions of small $M_{A}$ and large $\tan \beta$ [18]. At a future linear collider (ILC) a precise determination of the Higgs boson properties (either of the light Higgs boson at $\sim 125.6 \mathrm{GeV}$ or heavier MSSM Higgs bosons within the kinematic reach) will be possible [19]. In particular a mass measurement of the light Higgs boson with an accuracy below $\sim 0.05 \mathrm{GeV}$ is anticipated [20]. The interplay of the LHC and the ILC in the neutral MSSM Higgs sector has been discussed in Refs. [21-23]. 
For the $\operatorname{MSSM}^{1}$ the status of higher-order corrections to the masses and mixing angles in the neutral Higgs sector is quite advanced. The complete one-loop result within the MSSM is known [28-35]. The by far dominant one-loop contribution is the $\mathcal{O}\left(\alpha_{t}\right)$ term due to top and stop loops ( $\alpha_{t} \equiv h_{t}^{2} /(4 \pi), h_{t}$ being the top-quark Yukawa coupling). The computation of the two-loop corrections has meanwhile reached a stage where all the presumably dominant contributions are available [36-53]. In particular, the $\mathcal{O}\left(\alpha_{t} \alpha_{s}\right)$ contributions to the self-energies-evaluated in the Feynmandiagrammatic (FD) as well as in the effective potential (EP) method-as well as the $\mathcal{O}\left(\alpha_{t}^{2}\right), \mathcal{O}\left(\alpha_{b} \alpha_{s}\right), \mathcal{O}\left(\alpha_{t} \alpha_{b}\right)$ and $\mathcal{O}\left(\alpha_{b}^{2}\right)$ contributions-evaluated in the EP approach-are known for vanishing external momenta. An evaluation of the momentum dependence at the two-loop level in a pure $\overline{\mathrm{DR}}$ calculation was presented in Ref. [54]. A (nearly) full two-loop EP calculation, including even the leading threeloop corrections, has also been published [55-62]. However, within the EP method all contributions are evaluated at zero external momentum, in contrast to the FD method, which in principle allows non-vanishing external momentum. Further, the calculation presented in Refs. [55-62] is not publicly available as a computer code for Higgs-mass calculations. Subsequently, another leading three-loop calculation of $\mathcal{O}\left(\alpha_{t} \alpha_{s}^{2}\right)$, depending on the various SUSY mass hierarchies, has been performed [63-65], resulting in the code $\mathrm{H} 3 \mathrm{~m}$ (which adds the three-loop corrections to the FeynHiggs result). Most recently, a combination of the full one-loop result, supplemented with leading and subleading two-loop corrections evaluated in the Feynmandiagrammatic/effective potential method and a resummation of the leading and subleading logarithmic corrections from the scalar-top sector has been published [14] in the latest version of the code FeynHiggs [13,14,24,38,66,67]. While previous to this combination the remaining theoretical uncertainty on the lightest $\mathcal{C P}$-even Higgs boson mass had been estimated to be about $3 \mathrm{GeV}[12,13]$, the combined result was roughly estimated to yield an uncertainty of about $2 \mathrm{GeV}$ $[14,15]$; however, more detailed analyses will be necessary to yield a more solid result.

In the present paper we calculate the two-loop $\mathcal{O}\left(\alpha_{t} \alpha_{s}\right)$ corrections to the Higgs boson masses in a mixed onshell/DR scheme. Compared to previously known results [36-38,44] we evaluate here corrections that are proportional to the external momentum of the relevant Higgs boson selfenergies. These corrections can directly be added to the corrections included in FeynHiggs. An overview of the relevant sectors and the calculation is given in Sect. 2, whereas in Sect. 3 we discuss the size and relevance of the new two-loop corrections. Our conclusions are given in Sect. 4.

\footnotetext{
1 We concentrate here on the case with real parameters. For the case of complex parameters see Refs. [24-27] and references therein.
}

\section{Calculation}

\subsection{The Higgs-boson sector of the MSSM}

The MSSM requires two scalar doublets, which are conventionally written in terms of their components as follows:

$\mathcal{H}_{1}=\left(\begin{array}{c}\mathcal{H}_{1}^{0} \\ \mathcal{H}_{1}^{-}\end{array}\right)=\left(\begin{array}{c}v_{1}+\frac{1}{\sqrt{2}}\left(\phi_{1}^{0}-i \chi_{1}^{0}\right) \\ -\phi_{1}^{-}\end{array}\right)$,
$\mathcal{H}_{2}=\left(\begin{array}{c}\mathcal{H}_{2}^{+} \\ \mathcal{H}_{2}^{0}\end{array}\right)=\left(\begin{array}{c}\phi_{2}^{+} \\ v_{2}+\frac{1}{\sqrt{2}}\left(\phi_{2}^{0}+i \chi_{2}^{0}\right)\end{array}\right)$.

The Higgs boson sector can be described with the help of two independent parameters (besides the SM gauge couplings), conventionally chosen as $\tan \beta=v_{2} / v_{1}$, the ratio of the two vacuum expectation values, and $M_{A}^{2}$, the mass of the $\mathcal{C P}$-odd Higgs boson $A$. The bilinear part of the Higgs potential leads to the tree-level mass matrix for the neutral $\mathcal{C P}$-even Higgs boson,

$$
\begin{aligned}
& M_{\text {Higgs }}^{2, \text { tree }}=\left(\begin{array}{cc}
m_{\phi_{1}}^{2} & m_{\phi_{1} \phi_{2}}^{2} \\
m_{\phi_{1} \phi_{2}}^{2} & m_{\phi_{2}}^{2}
\end{array}\right) \\
& =\left(\begin{array}{cc}
M_{A}^{2} \sin ^{2} \beta+M_{Z}^{2} \cos ^{2} \beta & -\left(M_{A}^{2}+M_{Z}^{2}\right) \sin \beta \cos \beta \\
-\left(M_{A}^{2}+M_{Z}^{2}\right) \sin \beta \cos \beta & M_{A}^{2} \cos ^{2} \beta+M_{Z}^{2} \sin ^{2} \beta
\end{array}\right),
\end{aligned}
$$

in the $\left(\phi_{1}, \phi_{2}\right)$ basis and being expressed in terms of the parameters $M_{Z}, M_{A}$ and the angle $\beta$. Diagonalization yields the tree-level masses $m_{h \text {,tree, }} m_{H \text {,tree }}$.

The higher-order corrected $\mathcal{C P}$-even Higgs boson masses in the MSSM are obtained from the corresponding propagators dressed by their self-energies. The inverse propagator matrix in the $\left(\phi_{1}, \phi_{2}\right)$ basis is given by

$$
\begin{aligned}
& \left(\Delta_{\text {Higgs }}\right)^{-1} \\
& \quad=-\mathrm{i}\left(\begin{array}{ll}
p^{2}-m_{\phi_{1}}^{2}+\hat{\Sigma}_{\phi_{1}}\left(p^{2}\right) & -m_{\phi_{1} \phi_{2}}^{2}+\hat{\Sigma}_{\phi_{1} \phi_{2}}\left(p^{2}\right) \\
-m_{\phi_{1} \phi_{2}}^{2}+\hat{\Sigma}_{\phi_{1} \phi_{2}}\left(p^{2}\right) & p^{2}-m_{\phi_{2}}^{2}+\hat{\Sigma}_{\phi_{2}}\left(p^{2}\right)
\end{array}\right),
\end{aligned}
$$

where the $\hat{\Sigma}\left(p^{2}\right)$ denote the renormalized Higgs-boson selfenergies, $p$ being the external momentum. The renormalized self-energies can be expressed through the unrenormalized self-energies, $\Sigma\left(p^{2}\right)$, and counterterms involving renormalization constants $\delta m^{2}$ and $\delta Z$ from parameter and field renormalization. With the self-energies expanded up to two-loop order, $\hat{\Sigma}=\hat{\Sigma}^{(1)}+\hat{\Sigma}^{(2)}$, one has for the $\mathcal{C P}$-even part at the $i$-loop level $(i=1,2)$,

$$
\begin{gathered}
\hat{\Sigma}_{\phi_{1}}^{(i)}\left(p^{2}\right)=\Sigma_{\phi_{1}}^{(i)}\left(p^{2}\right)+\delta Z_{\phi_{1}}^{(i)}\left(p^{2}-m_{\phi_{1}}^{2}\right)-\delta m_{\phi_{1}}^{2(i)}, \\
\hat{\Sigma}_{\phi_{2}}^{(i)}\left(p^{2}\right)=\Sigma_{\phi_{2}}^{(i)}\left(p^{2}\right)+\delta Z_{\phi_{2}}^{(i)}\left(p^{2}-m_{\phi_{2}}^{2}\right)-\delta m_{\phi_{2}}^{2(i)}, \\
\hat{\Sigma}_{\phi_{1} \phi_{2}}^{(i)}\left(p^{2}\right)=\Sigma_{\phi_{1} \phi_{2}}^{(i)}\left(p^{2}\right)-\delta Z_{\phi_{1} \phi_{2}}^{(i)} m_{\phi_{1} \phi_{2}}^{2}-\delta m_{\phi_{1} \phi_{2}}^{2(i)} .
\end{gathered}
$$


The counterterms are determined by appropriate renormalization conditions and are given in the appendix.

The renormalized self-energies in the $\left(\phi_{1}, \phi_{2}\right)$ basis can be rotated into the physical $(h, H)$ basis where the tree-level propagator matrix is diagonal, via

$$
\left(\begin{array}{cc}
\hat{\Sigma}_{H H} & \hat{\Sigma}_{h H} \\
\hat{\Sigma}_{h H} & \hat{\Sigma}_{h h}
\end{array}\right)=D(\alpha)\left(\begin{array}{cc}
\hat{\Sigma}_{\phi_{1}} & \hat{\Sigma}_{\phi_{1} \phi_{2}} \\
\hat{\Sigma}_{\phi_{1} \phi_{2}} & \hat{\Sigma}_{\phi_{2}}
\end{array}\right) D^{T}(\alpha)
$$

with the matrix

$$
D(\alpha)=\left(\begin{array}{cc}
\cos \alpha & \sin \alpha \\
-\sin \alpha & \cos \alpha
\end{array}\right),
$$

which diagonalizes the tree-level mass matrix (2). The $\mathcal{C P}$ even Higgs boson masses are determined by the poles of the $(h, H)$-propagator matrix. This is equivalent to solving the equation

$$
\begin{aligned}
& {\left[p^{2}-m_{h, \text { tree }}^{2}+\hat{\Sigma}_{h h}\left(p^{2}\right)\right]\left[p^{2}-m_{H, \text { tree }}^{2}+\hat{\Sigma}_{H H}\left(p^{2}\right)\right]} \\
& \quad-\left[\hat{\Sigma}_{h H}\left(p^{2}\right)\right]^{2}=0,
\end{aligned}
$$

yielding the loop-corrected pole masses, $M_{h}$ and $M_{H}$. Here we use the implementation in the code FeynHiggs $[13,14,24,38,66,67]$, supplemented by the new momentumdependent $\mathcal{O}\left(\alpha_{t} \alpha_{S}\right)$ corrections, as described in Sect. 2.4.

Our calculation is performed in the Feynman-diagrammatic (FD) approach. To arrive at expressions for the unrenormalized self-energies and tadpoles at $\mathcal{O}\left(\alpha_{t} \alpha_{s}\right)$, the evaluation of genuine two-loop diagrams and one-loop graphs with counterterm insertions is required. Example diagrams for the neutral Higgs-boson self-energies are shown in Fig. 1, and for the tadpoles in Fig. 2. For the counterterm insertions, described in Sect. 2.2, one-loop diagrams with external top quarks/squarks have to be evaluated as well, as displayed in Fig. 3. The complete set of contributing Feynman diagrams has been generated with the program FeynArts [68-71] (using the model file including counterterms from Ref. [72]), tensor reduction and the evaluation of traces was done with support from the programs FormCalc [73] and TwoCalc [74,75], yielding algebraic expressions in terms of the scalar one-loop functions $A_{0}, B_{0}$ [76], the massive vacuum two-loop functions [77], and two-loop integrals which depend on the external momentum. These integrals have been evaluated with the program SecDec [78, 79]; see Sect. 2.3.

\subsection{The scalar-top sector of the MSSM}

The bilinear part of the top-squark Lagrangian,

$$
\mathcal{L}_{\tilde{t}, \text { mass }}=-\left(\tilde{t}_{L}^{\dagger}, \tilde{t}_{R}^{\dagger}\right) \mathbf{M}_{\tilde{t}}\left(\begin{array}{c}
\tilde{t}_{L} \\
\tilde{t}_{R}
\end{array}\right),
$$

contains the stop mass matrix $\mathbf{M}_{\tilde{t}}$, given by
$\mathbf{M}_{\tilde{t}}=\left(\begin{array}{cc}M_{\tilde{t}_{L}}^{2}+m_{t}^{2}+M_{Z}^{2} \cos 2 \beta\left(T_{t}^{3}-Q_{t} s_{\mathrm{W}}^{2}\right) & m_{t} X_{t} \\ m_{t} X_{t} & M_{\tilde{t}_{R}}^{2}+m_{t}^{2}+M_{Z}^{2} \cos 2 \beta Q_{t} s_{\mathrm{w}}^{2}\end{array}\right)$,

with

$X_{t}=A_{t}-\mu \cot \beta$.

$Q_{t}$ and $T_{t}^{3}$ denote the charge and isospin of the top quark, $A_{t}$ is the trilinear coupling between the Higgs bosons and the scalar tops, and $\mu$ is the Higgsino mass parameter. Below we use $M_{\text {SUSY }}:=M_{\tilde{t}_{L}}=M_{\tilde{t}_{R}}$ for our numerical evaluation. However, the analytical calculation has been performed for arbitrary $M_{\tilde{t}_{L}}$ and $M_{\tilde{t}_{R}}$. $\mathbf{M}_{\tilde{t}}$ can be diagonalized with the help of a unitary transformation matrix $\mathbf{U}_{\tilde{t}}$, parametrized by a mixing angle $\theta_{\tilde{t}}$, to provide the eigenvalues $m_{\tilde{t}_{1}}^{2}$ and $m_{\tilde{t}_{2}}^{2}$ as the squares of the two on-shell top-squark masses.

For the evaluation of the $\mathcal{O}\left(\alpha_{t} \alpha_{s}\right)$ two-loop contributions to the self-energies and tadpoles of the Higgs sector, renormalization of the top/stop sector at $\mathcal{O}\left(\alpha_{s}\right)$ is required, giving rise to the counterterms for one-loop subrenormalization (see Figs. 1,2). We follow the renormalization at the one-loop level given in Refs. [40,80-82], where details can be found. In the context of this paper, we only want to emphasize that on-shell (OS) renormalization is performed for the top-quark mass as well as for the scalar-top masses. This is different from the approach pursued, for example, in Ref. [54], where a $\overline{\mathrm{DR}}$ renormalization has been employed. Using the OS scheme allows us to consistently combine our new correction terms with the hitherto available self-energies included in FeynHiggs.

Finally, at $\mathcal{O}\left(\alpha_{t} \alpha_{s}\right)$, gluinos appear as virtual particles only at the two-loop level (hence, no renormalization for the gluinos is needed). The corresponding soft-breaking gluino mass parameter $M_{3}$ determines the gluino mass, $M_{\tilde{g}}=M_{3}$.

\subsection{The program SecDec}

The calculation of the momentum-dependent two-loop corrections to the Higgs-boson masses at order $\mathcal{O}\left(\alpha_{t} \alpha_{s}\right)$ involves two-loop two-point functions with up to four different masses, in addition to the mass scale given by the external momentum $p^{2}$. For two-loop diagrams of propagator type, analytical results in four space-time dimensions are known only sparsely if different masses are occurring in the loops [77,83-90]. The integrals which are lacking analytical results can be classified into four different topologies, shown in Fig. 4. We have calculated these integrals numerically using the program SecDec $[78,79]$, where up to four different masses in 34 different mass configurations needed to be considered, with differences in the kinematic invariants of several orders of magnitude.

The program SecDec is a publicly available tool [91] to calculate multi-loop integrals numerically. Dimensionally 
Fig. 1 Generic two-loop diagrams and diagrams with counterterm insertions for the Higgs-boson self-energies $(\phi=h, H, A)$

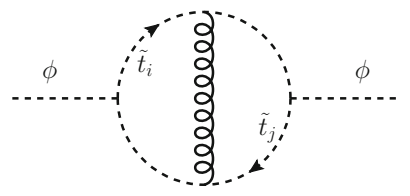

(a)

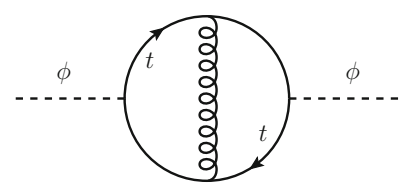

(b)

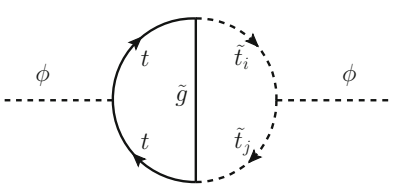

(c)

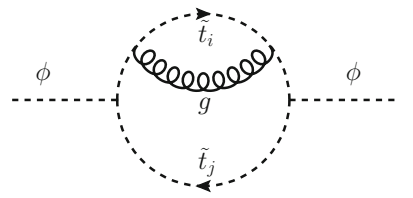

(d)

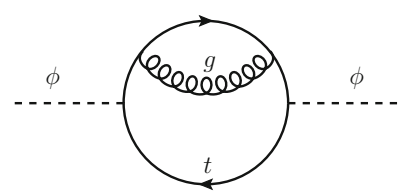

(e)

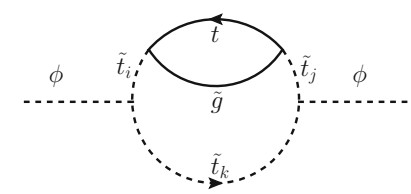

(f)

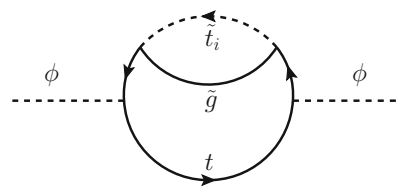

(g)

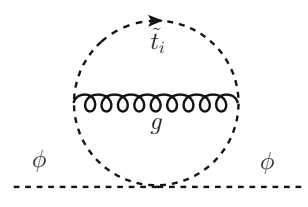

(h)

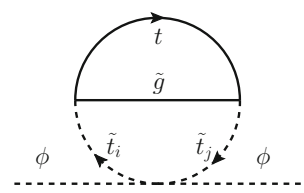

(i)

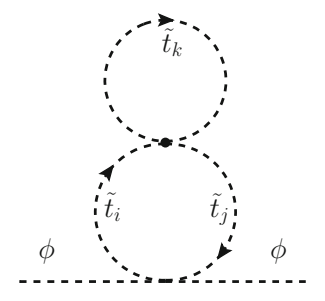

(j)

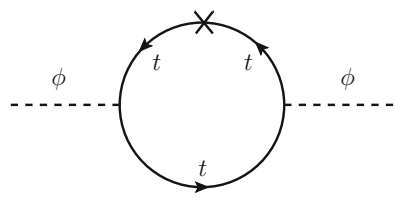

(m)

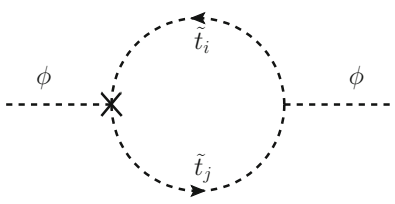

(p)

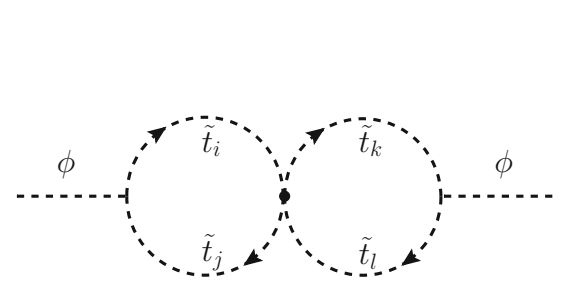

(k)

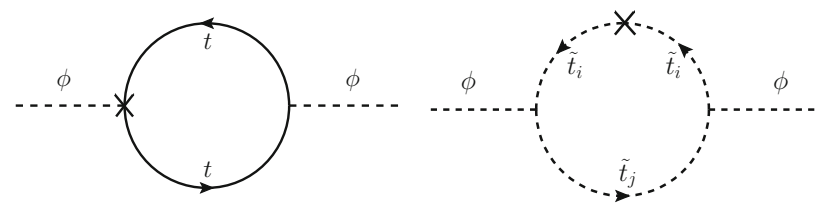

(n)

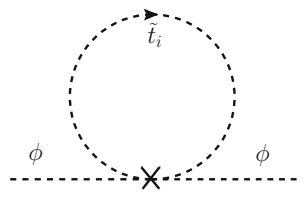

(q)

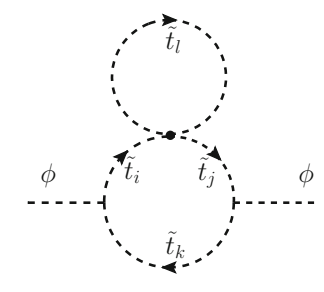

(l) (o)

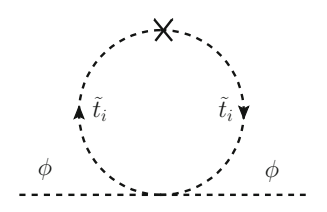

(r) regulated poles are factorized by sector decomposition as described in Refs. [92,93], while kinematic thresholds are handled by a deformation of the integration contour into the complex plane, as described e.g. in Ref. [79]. The numerical integration is done using the CUBA library [94].

The program has also been extended to be able to calculate tensor integrals of any rank [95], and to process efficiently the evaluation of large ranges of kinematic points using the "mult inumerics" feature of the program, which is of particular importance for the calculation presented here. This feature allows one to produce input files for large sets of kinematic points automatically, and to process the evaluation of these points in parallel if several cores or a cluster are available, without repeating the algebraic part of the sector 
Fig. 2 Generic two-loop diagrams and diagrams with counterterm insertions for the Higgs-boson tadpoles $(\phi=h, H ; i, j, k=1,2)$

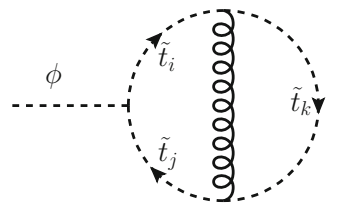

(a)

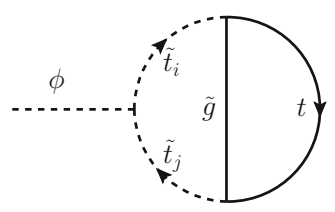

(b)

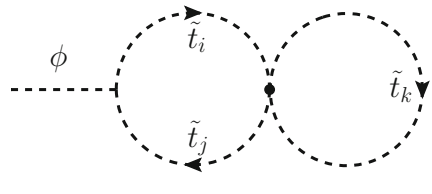

(c)

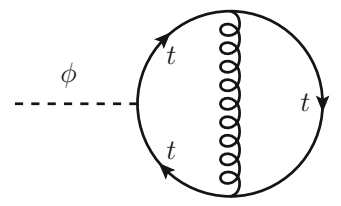

(d)

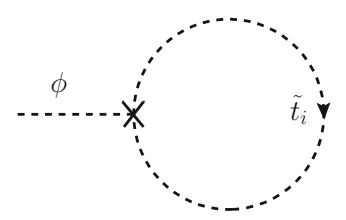

(f)

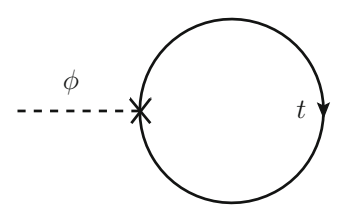

(h)

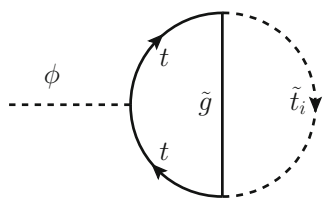

(e)

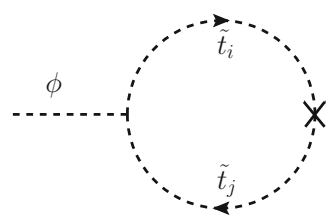

(g)

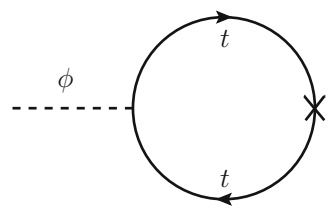

(i)

Fig. 3 Generic one-loop diagrams for subrenormalization counterterms, involving top quarks $t$, top squarks $\tilde{t}$, gluons $g$ and gluinos $\tilde{g}(i, j, k=1,2)$

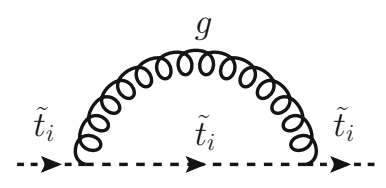

(a)

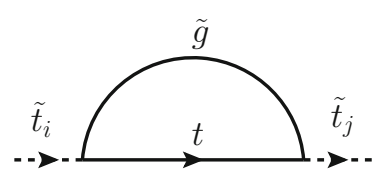

(c)

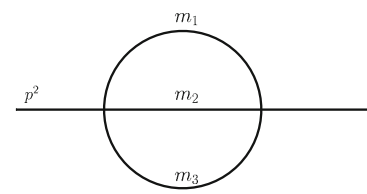

$T_{234}$

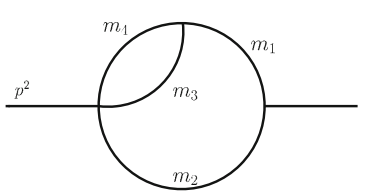

$T_{1234}$

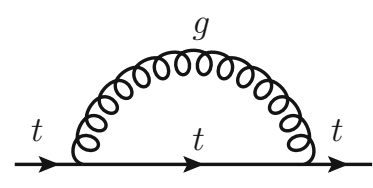

(b)

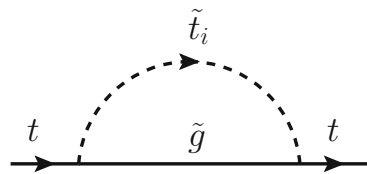

(d)

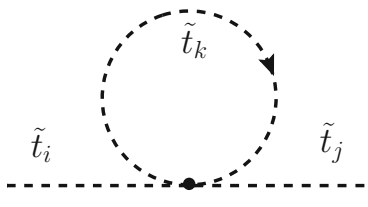

(e)

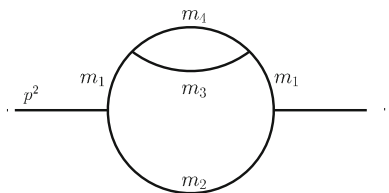

$T_{11234}$

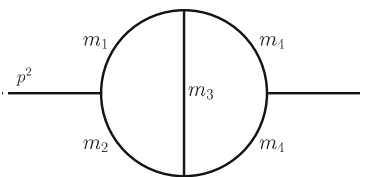

$T_{12345}$

Fig. 4 Topologies which have been calculated numerically using SecDec 
decomposition, which can be done once and for all. The evaluation of a single phase space point for the most complicated topology, to reach a relative accuracy of at least $10^{-5}$, ranges between 0.01 and $100 \mathrm{~s}$ on an Intel core i7 processor, where the larger timings are for points very close to a kinematic threshold.

\subsection{Evaluation and implementation in the program FeynHiggs}

The resulting new contributions to the neutral $\mathcal{C P}$-even Higgs-boson self-energies, containing all momentum-dependent and additional constant terms, are assigned to the differences

$\Delta \hat{\Sigma}_{a b}^{(2)}\left(p^{2}\right)=\hat{\Sigma}_{a b}^{(2)}\left(p^{2}\right)-\tilde{\Sigma}_{a b}^{(2)}(0), \quad a b=\{H H, h H, h h\}$.

Note the tilde (not hat) on $\tilde{\Sigma}^{(2)}(0)$ which signifies that not only the self-energies are evaluated at zero external momentum but also the corresponding counterterms, following Refs. [36-38]. A finite shift $\Delta \hat{\Sigma}^{(2)}(0)$ therefore remains in the limit $p^{2} \rightarrow 0$ due to $\delta M_{A}^{2(2)}=\operatorname{Re} \Sigma_{A A}^{(2)}\left(M_{A}^{2}\right)$ being computed at $p^{2}=M_{A}^{2}$ in $\hat{\Sigma}^{(2)}$, but at $p^{2}=0$ in $\tilde{\Sigma}^{(2)}$; for details see Eqs. (22) and (24) in the appendix.

The numerical evaluation to derive the physical masses for $h, H$ as the poles (real parts) of the dressed propagators proceeds on the basis of Eq. (7) in an iterative way.

- In a first step, the squared masses $M_{h, 0}^{2}, M_{H, 0}^{2}$ are determined by solving Eq. (7) excluding the new terms $\Delta \hat{\Sigma}_{a b}^{(2)}\left(p^{2}\right)$ from the self-energies.

- In a second step, the shifts $\Delta \hat{\Sigma}_{a b}^{(2)}\left(M_{h, 0}^{2}\right) \equiv c_{a b}^{h}$ and $\Delta \hat{\Sigma}_{a b}^{(2)}\left(M_{H, 0}^{2}\right) \equiv c_{a b}^{H}$ are calculated and added as constants to the self-energies in Eq. (7), $\hat{\Sigma}_{a b}\left(p^{2}\right) \rightarrow$ $\hat{\Sigma}_{a b}\left(p^{2}\right)+c_{a b}^{h(H)}$

- In the third step, Eq. (7) is solved again, now including the constant shifts $c_{a b}^{h(H)}$ in the self-energies, to deliver the refined masses $M_{h}$ (with $c_{a b}^{h}$ ) and $M_{H}$ (with $c_{a b}^{H}$ ).

This procedure can be repeated for improving the accuracy; numerically it turns out that going beyond the first iteration yields only marginal changes.

The corrections of Eq. (11) are incorporated in FeynHiggs by the following recipe, which is more general and in principle applicable also to the case of the complex MSSM with $\mathcal{C P}$ violation.

1. Determine Higgs masses $M_{h_{i}, 0}$ without the momentumdependent terms of Eq. (11); the index $i=1, \ldots, 4$ enumerates the masses of $h, H, A, H^{ \pm}$in the real MSSM. This is done by invoking the FeynHiggs mass-finder.
2. Compute the shifts $c_{a b}^{h_{k}}=\Delta \hat{\Sigma}_{a b}^{(2)}\left(M_{h_{k}, 0}^{2}\right)$ with $a, b, h_{k}=$ $h, H$.

3. Run FeynHiggs' mass-finder again including the $c_{a b}^{h_{k}}$ as constant shifts in the self-energies to determine the refined Higgs masses $M_{h}$ and $M_{H}$.

This procedure could conceivably be iterated until full selfconsistency is reached; yet the resulting mass improvements turn out to be too small to justify extra CPU time.

On the technical side we added an interface for an external program to FeynHiggs which exports relevant model parameters to the external program's environment, currently:

$\begin{array}{llll}\text { FHscalefactor } & \text { ren. scale multiplicator, } & \text { FHTB } & \tan \beta, \\ \text { FHAlfasmT } & \alpha_{s}\left(m_{t}\right), & \text { FHGF } & G_{F}, \\ \text { FHMHiggs } 2 i & M_{h_{i}, 0}^{2}, i=1 \ldots 4, & \text { FHMSt } i & m_{\tilde{t}, i}, i=1,2, \\ \text { FH\{Re, Im\}USt } 1 i & U_{\tilde{t}, 1 i}, i=1,2, & \text { FHMGl } & m_{\tilde{g}}, \\ \text { FH\{Re, Im }\} \text { MUE } & \mu, & \text { FHMA0 } & M_{A},\end{array}$

where the $U_{\tilde{t}, 1 i}$ denote the elements of the stop mixing matrix, $\alpha_{s}\left(m_{t}\right)$ the running strong coupling at the scale $m_{t}$, and $G_{F}$ the Fermi constant. The renormalization scale is defined within FeynHiggs as $\mu_{R}=m_{t} \cdot$ FHscalefactor. Invocation of the external program is switched on by providing its path in the environment variable FHEXTSE. The program is executed from inside a temporary directory which is afterwards removed.

The output (stdout) is scanned for lines of the form 'se@m $c_{r} c_{i}$ ' which specify the correction $c_{r}+\mathrm{i} c_{i}$ [with $\left.c_{r}=\operatorname{Re}\left(c_{a b}^{h_{k}}\right), c_{i}=\operatorname{Im}\left(c_{a b}^{h_{k}}\right)\right]$ to self-energy se in the computation of mass $m$, where $m$ is one of Mh0, MHH, MAO, MHp, and se is one of hOhO, HHHH, AOAO, HmHp, hOHH, hOAO, HHA0, G0G0, h0G0, HHG0, A0G0, GmGp, HmGp, F1F1, F2F2, F1F2. The latter three, if given, substitute

$\mathrm{HHHH}=\cos ^{2} \alpha \mathrm{F} 1 \mathrm{~F} 1+\sin ^{2} \alpha \mathrm{F} 2 \mathrm{~F} 2+\sin 2 \alpha \mathrm{F} 1 \mathrm{~F} 2$,

h0h0 $=\sin ^{2} \alpha \mathrm{F} 1 \mathrm{~F} 1+\cos ^{2} \alpha \mathrm{F} 2 \mathrm{~F} 2-\sin 2 \alpha \mathrm{F} 1 \mathrm{~F} 2$,

$\mathrm{h} 0 \mathrm{HH}=\cos 2 \alpha \mathrm{F} 1 \mathrm{~F} 2+\frac{1}{2} \sin 2 \alpha(\mathrm{F} 2 \mathrm{~F} 2-\mathrm{F} 1 \mathrm{~F} 1)$,

where $\alpha$ is the tree-level $2 \times 2$ neutral-Higgs mixing angle in Eq. (6). Self-energies not given are assumed zero.

The zero-momentum contributions $\tilde{\Sigma}_{a b}^{(2)}(0), a b=\{H H$, $h H, h h\}$, are subtracted if the output of the external program contains one or more of 'sub asat', 'sub atat', 'sub asab', 'sub atab' for the $\alpha_{s} \alpha_{t}, \alpha_{t}^{2}, \alpha_{s} \alpha_{b}$, and $\alpha_{t} \alpha_{b}$ contributions, respectively. All other lines in the output are ignored. 

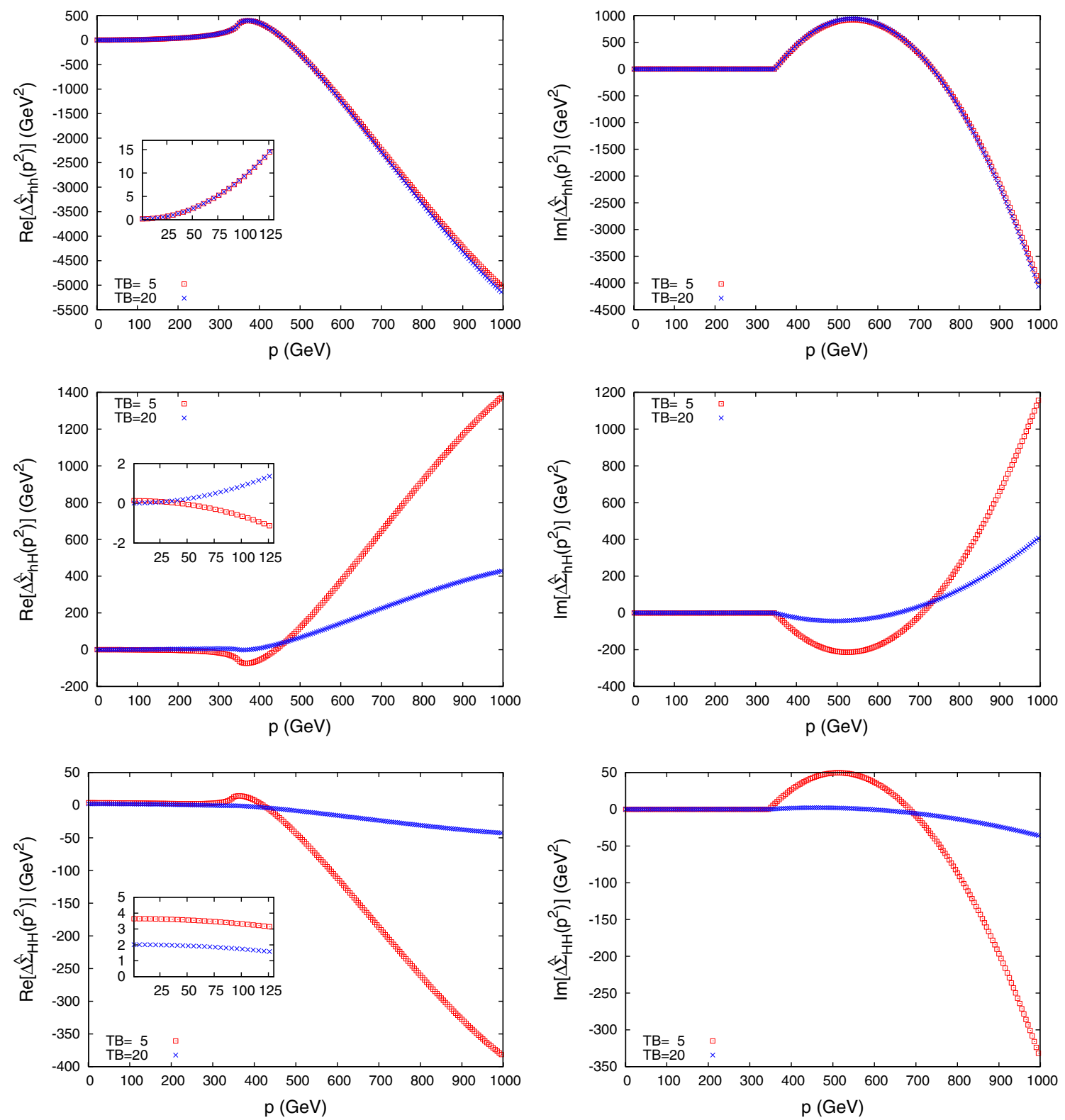

Fig. 5 Momentum dependence of the real (left column) and imaginary (right column) parts of the two-loop self-energies $\Delta \hat{\Sigma}_{h h}, \Delta \hat{\Sigma}_{h H}, \Delta \hat{\Sigma}_{H H}$, within scenario 1 , for $\tan \beta=5$ (red squares)

\section{Numerical results}

We show results for the subtracted two-loop self-energies $\Delta \hat{\Sigma}_{a b}^{(2)}\left(p^{2}\right)$ given in Eq. (11), as well as for the mass shifts

$$
\Delta M_{h}=M_{h}-M_{h, 0}, \quad \Delta M_{H}=M_{H}-M_{H, 0}
$$

i.e. the difference in the physical Higgs-boson masses evaluated including and excluding the newly obtained momentumdependent two-loop corrections. This quantity, in particular $\Delta M_{h}$ for the light $\mathcal{C P}$-even Higgs boson, can directly be compared with the current experimental uncertainty as well

and $\tan \beta=20$ (blue crosses) and $M_{A}=250 \mathrm{GeV}$. One can see that the self-energies change substantially beyond the threshold at $p^{2}=\left(2 m_{t}\right)^{2}$

as with the anticipated future ILC accuracy of [20],

$\delta M_{h}^{\text {exp,ILC }} \lesssim 0.05 \mathrm{GeV}$

The results are obtained for two different scenarios, varying parameters like $\tan \beta, M_{A}, M_{\tilde{g}}$, and illustrate the impact of these parameters via the new two-loop corrections on the neutral $\mathcal{C} \mathcal{P}$-even Higgs boson masses, $M_{h}$ and $M_{H}$. The corresponding renormalization scale, $\mu_{\overline{\mathrm{DR}}}$, is set to $\mu_{\overline{\mathrm{DR}}}=m_{t}$ in all numerical evaluations. The scale uncertainties are expected to be much smaller than the parametric uncertainties due to variations of parameters like $\tan \beta, M_{A}, M_{\tilde{g}}, m_{\tilde{t}}$. 

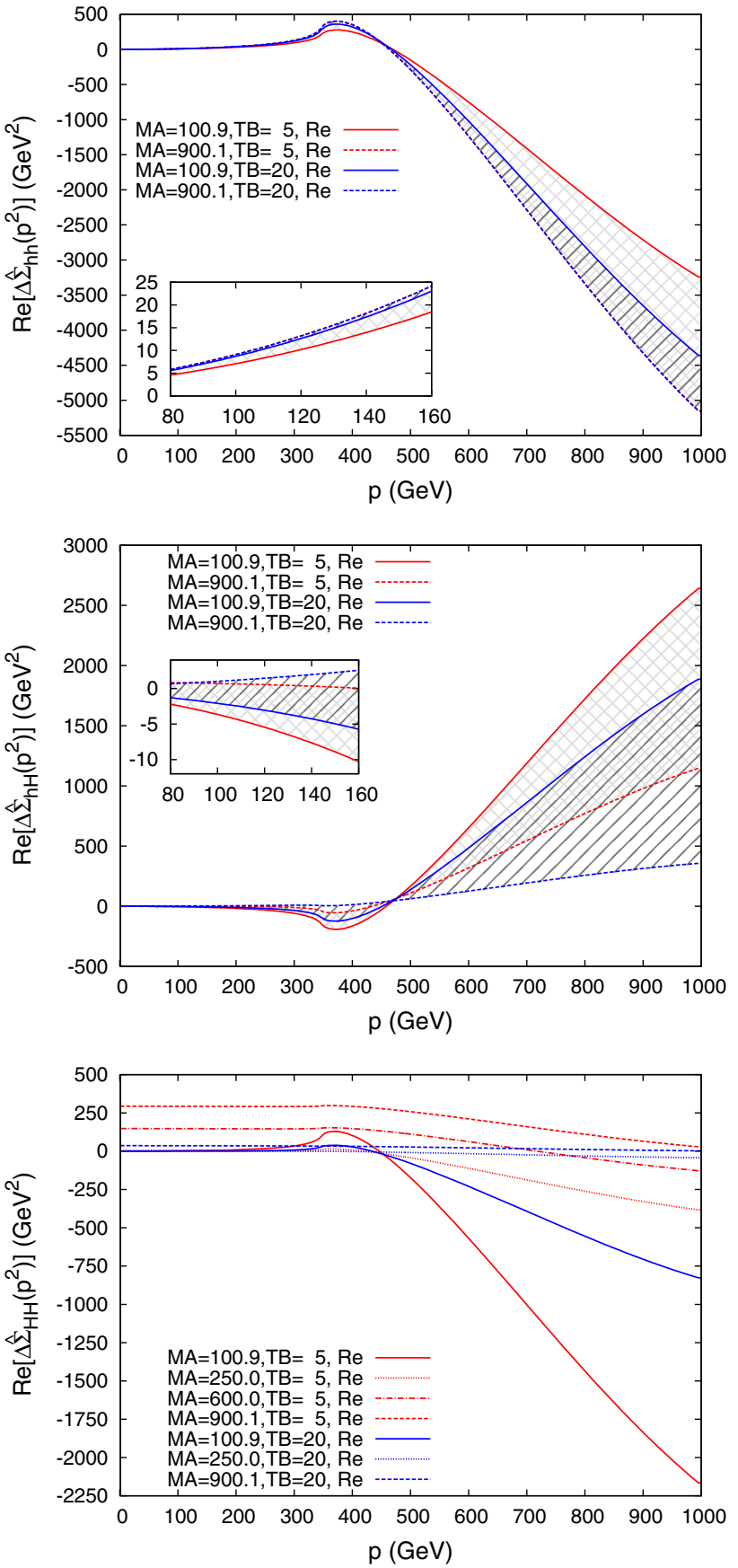

Fig. 6 Momentum dependence of the real part of the two-loop selfenergies $\Delta \hat{\Sigma}_{h h}, \Delta \hat{\Sigma}_{h H}, \Delta \hat{\Sigma}_{H H}$, within scenario 1 , for two different values of $\tan \beta$ and a range of $M_{A}$ values

\subsection{Scenario $1: m_{h}^{\max }$}

Scenario 1 is oriented at the $m_{h}^{\max }$ scenario described in Ref. [96]. We use the following parameters:

$m_{t}=173.2 \mathrm{GeV}, \quad M_{\mathrm{SUSY}}=1 \mathrm{TeV}, \quad X_{t}=2 M_{\mathrm{SUSY}}$,

$M_{\tilde{g}}=1500 \mathrm{GeV}, \quad \mu=200 \mathrm{GeV}$,
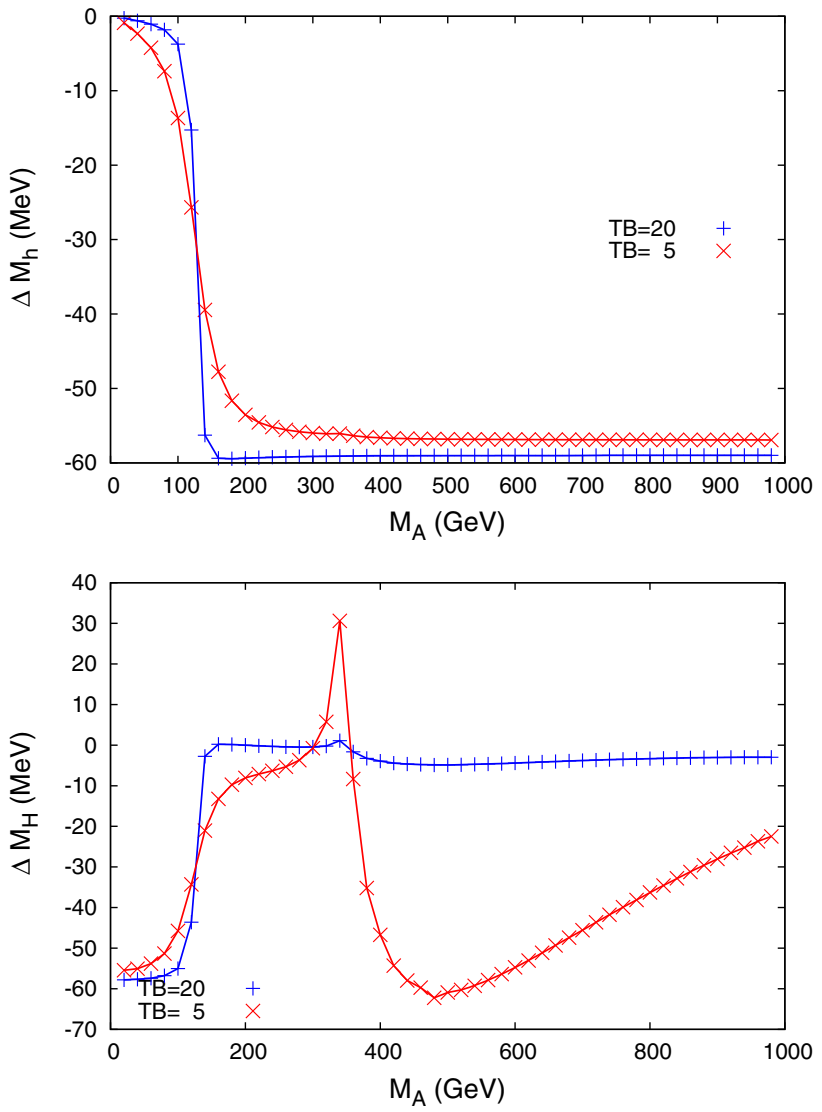

Fig. 7 Variation of the mass shifts $\Delta M_{h}, \Delta M_{H}$ with the $A$-boson mass $M_{A}$ within scenario 1 , for $\tan \beta=5$ (red) and $\tan \beta=20$ (blue). The peak in $\Delta M_{H}$ originates from a threshold at $2 m_{t}$

leading to stop mass values of

$m_{\tilde{t}_{1}}=826.8 \mathrm{GeV}, \quad m_{\tilde{t}_{2}}=1173.2 \mathrm{GeV}$.

With the introduction of the momentum dependence, thresholds occur in the self-energy diagrams when the external momentum $p=\sqrt{p^{2}}$, in the time-like region, is such that a cut of the diagram would correspond to on-shell production of the massive particles of the cut propagators. The resulting imaginary parts enter in the search for the complex poles of the inverse propagator matrix of the Higgs bosons. Therefore it is interesting to study the behavior of the real and imaginary parts of the self-energies. In Fig. 5 we show the momentum-dependent parts of the renormalized two-loop self-energies in the physical basis, Eq. (11) for two different values of $\tan \beta, \tan \beta=5$ and $\tan \beta=20$, at a fixed $A$-boson mass $M_{A}=250 \mathrm{GeV}$. The data points are not connected by a line in order to show that each numerical point is obtained from a calculation of the 34 analytically unknown integrals with the program SecDec. The inlays in Fig. 5 magnify the region $p^{2} \leq(125 \mathrm{GeV})^{2}$, where one can observe that for $p^{2} \rightarrow 0$, the subtracted self-energies are not exactly zero. As mentioned in Sect. 2.4, this is due to the fact 

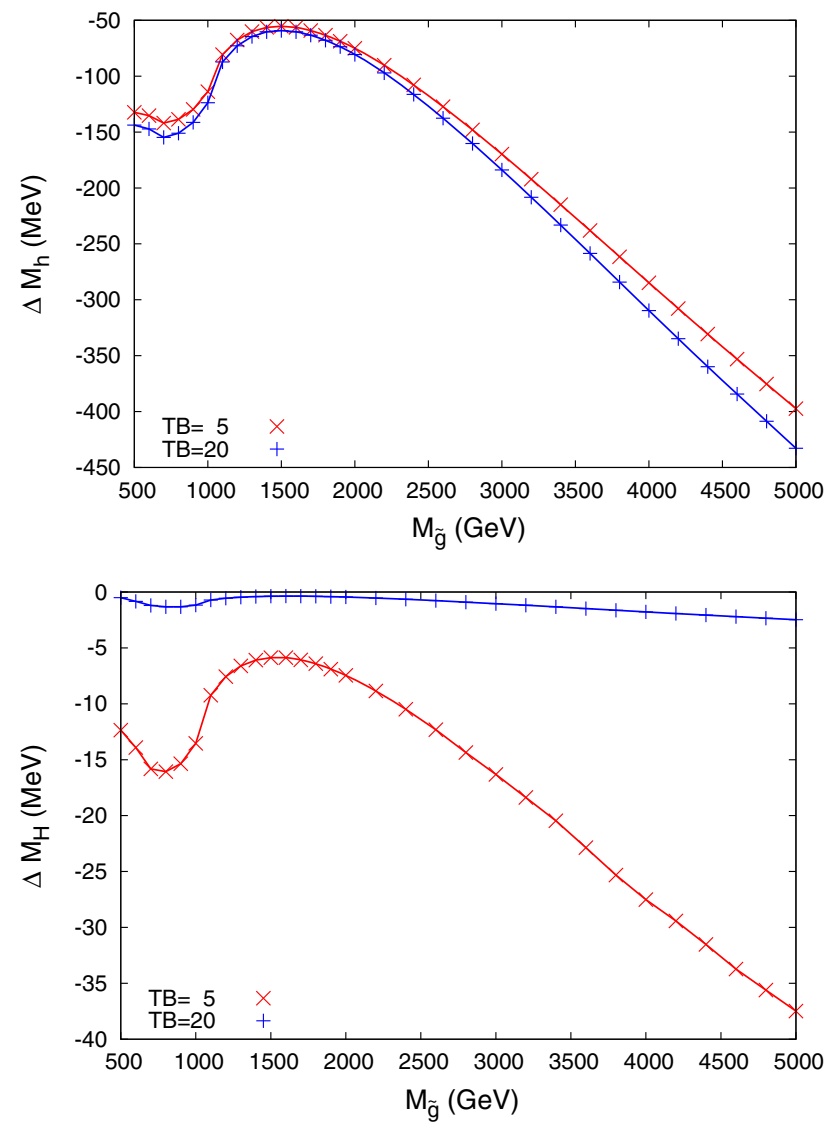

Fig. 8 Variation of the mass shifts $\Delta M_{h}, \Delta M_{H}$ with the gluino mass, within scenario 1 , for two different values of $\tan \beta=5,20$ and $M_{A}=$ $250 \mathrm{GeV}$

that the on-shell renormalization condition for the $A$-boson self-energy is defined differently with regard to the calculation without momentum dependence. The resulting constant contributions are additionally suppressed by factors $\sin ^{2} \beta$, $\sin \beta \cos \beta$ and $\cos ^{2} \beta$ appearing in the counter terms $\delta m_{\phi_{1}}^{2(2)}$, $\delta m_{\phi_{1} \phi_{2}}^{2(2)}$ and $\delta m_{\phi_{2}}^{2(2)}$, respectively, according to Eqs. (24) in the appendix.

The imaginary part is independent of the $A$-boson mass, as this mass parameter solely appears in the counterterms of $\overline{\mathrm{DR}}$ renormalized quantities and the $\delta M_{A}^{2(2)}$ counterterm, where only the real part contributes. Therefore, the imaginary parts displayed in Fig. 5 do not contain additional constant terms. As to be expected, the imaginary parts are zero below the $t \bar{t}$ production threshold at $p=2 m_{t}$, which results from the fact that the top mass is the smallest mass appearing in the loops. Beyond this threshold, the imaginary parts are growing substantially with increasing $p^{2}$. From these observations, the mass shifts in the region below the first threshold at $p=$ $2 m_{t}$ are expected not to be large.

Similar results, now including a variation of $M_{A}$ are shown in Fig. 6. In the upper plot for $\Delta \hat{\Sigma}_{h h}$ and in the middle plot for $\Delta \hat{\Sigma}_{h H}$ the solid lines depict $M_{A} \sim 100 \mathrm{GeV}$, while the

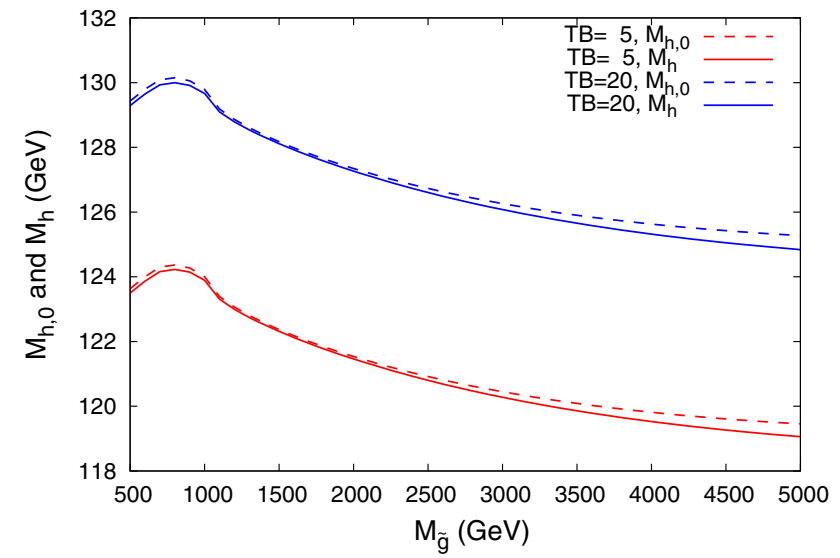

Fig. 9 Variation of $M_{h}$ and $M_{h, 0}$ as a function of $M_{\tilde{g}}$ within scenario 1 , for $\tan \beta=5,20$ and $M_{A}=250 \mathrm{GeV}$

dashed lines are for $M_{A} \sim 900 \mathrm{GeV}$. In these plots the light shading covers the range for $\tan \beta=5$, while the dark shading for $\tan \beta=20$. In the lower plot for $\Delta \hat{\Sigma}_{H H}$ we show results for $M_{A} \sim 100,250,600,900 \mathrm{GeV}$ as solid, dotted, dot-dashed, dashed lines, respectively (and shading has been omitted). For $\Delta \hat{\Sigma}_{h h}$ at low $p$ values only a small variation with $M_{A}$ can be observed. For $p$ and $M_{A}$ large, the contributions to the self-energy are bigger. In $\Delta \hat{\Sigma}_{h H}$ larger effects are observed at smaller $M_{A}$ for both small and large $p$ values. For $\Delta \hat{\Sigma}_{H H}$, on the other hand, at low $p$ values, large effects can be observed for large $M_{A}$ due to the aforementioned counterterm contribution $\sim \delta M_{A}^{2(2)}=\operatorname{Re} \Sigma_{A A}^{(2)}\left(M_{A}^{2}\right)$. At large $p$, as before, small $M_{A}$ values give a more sizable contribution.

We now turn to the effects of our newly computed momentum-dependent two-loop corrections on the Higgsboson masses $M_{h, H}$ via the mass shifts $\Delta M_{h}$ and $\Delta M_{H}$. In Fig. 7 we show $\Delta M_{h}$ (upper plot) and $\Delta M_{H}$ (lower plot) as a function of $M_{A}$ for $\tan \beta=5$ (blue) and $\tan \beta=20$ (red). In the $m_{h}^{\max }$ scenario for $M_{A} \gtrsim 200 \mathrm{GeV}$ we find $\Delta M_{h} \sim-60 \mathrm{MeV}$, i.e. of the size of the future experimental precision; see Eq. (14). The contribution to the heavy $\mathcal{C P}$-even Higgs-boson is suppressed with $\tan \beta$. While the size of $\Delta M_{H}$ becomes negligible for $M_{A} \gtrsim 150 \mathrm{GeV}$ for $\tan \beta=20$, its variation is more pronounced for $\tan \beta=5$. $\Delta M_{H}$ can reach about $-60 \mathrm{MeV}$ for very small or intermediate values of $M_{A}$ and steadily decreases for $M_{A} \gtrsim 500 \mathrm{GeV}$. The peak in $\Delta M_{H}$ for $\tan \beta=5$ originates from a threshold at $2 m_{t}$.

Finally, in scenario 1, we analyze the dependence of $M_{h}$ and $M_{H}$ on the gluino mass, $M_{\tilde{g}}$. The results are shown in Fig. 8 for $\Delta M_{h}$ (upper plot) and $\Delta M_{H}$ (lower plot) for $M_{A}=250 \mathrm{GeV}$, with the same color coding as in Fig. 7 . In the upper plot one can observe that the effects are particularly small for the default value of $M_{\tilde{g}}$ in scenario 1 . More sizeable shifts occur for larger gluino masses, by more 

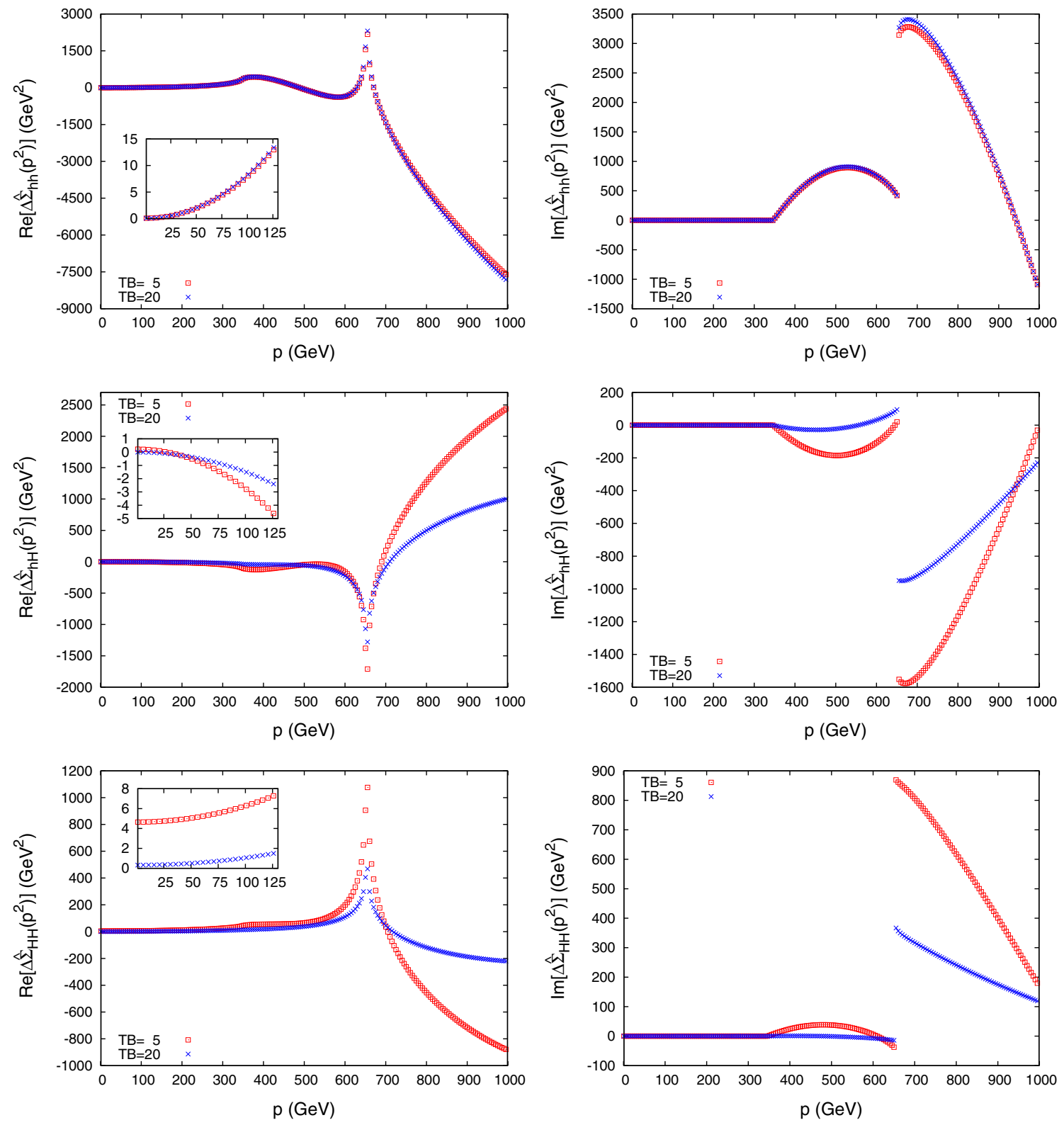

Fig. 10 Momentum dependence of the real and imaginary parts of the two-loop self-energies $\Delta \hat{\Sigma}_{h h}, \Delta \hat{\Sigma}_{h H}, \Delta \hat{\Sigma}_{H H}$ within scenario 2 , with $\tan \beta=5,20$ and $M_{A}=250 \mathrm{GeV}$ with the same color coding as in Fig. 5

than $-400 \mathrm{MeV}$ for $M_{\tilde{g}} \gtrsim 4 \mathrm{TeV}$, reaching thus the level of the current experimental accuracy in the Higgs-boson mass determination. The corrections to $M_{H}$, for the given value of $M_{A}=250 \mathrm{GeV}$ do not exceed $-50 \mathrm{MeV}$ in the considered $M_{\tilde{g}}$ range.

The dependence of the light $\mathcal{C} \mathcal{P}$-even Higgs-boson mass on $M_{\tilde{g}}$ is analyzed in Fig. 9 for $\tan \beta=5,20$ and $M_{A}=$ $250 \mathrm{GeV}$. Here we show as dashed lines the results for $M_{h, 0}$ (i.e. without the newly obtained momentum-dependent twoloop corrections) and as solid lines the results for $M_{h}$ (i.e. including the new corrections). While a maximum of the Higgs-boson mass can be observed around $M_{\tilde{g}} \sim 800 \mathrm{GeV}$, in agreement with the original definition of the $m_{h}^{\max }$ scenario [97], a downward shift by more than $4 \mathrm{GeV}$ is found for $M_{\tilde{g}} \sim 5 \mathrm{TeV}$. Such a strong effect is due to a (squared) logarithmic dependence of the $\mathcal{O}\left(\alpha_{t} \alpha_{s}\right)$ corrections evaluated at $p^{2}=0$, as given in Eq. (73) of Ref. [38]. In Fig. 9 it can be seen that the size of the momentum-dependent two-loop corrections similarly grows with $M_{\tilde{g}}$, reaching $\sim 400 \mathrm{MeV}$, as was shown above in Fig. 8. Consequently, the logarithmic dependence of the light $\mathcal{C P}$-even Higgs-boson mass on the gluino mass that was found analytically for the $\mathcal{O}\left(\alpha_{t} \alpha_{s}\right)$ corrections at $p^{2}=0$, is now also found numerically for the momentum-dependent two-loop corrections. 

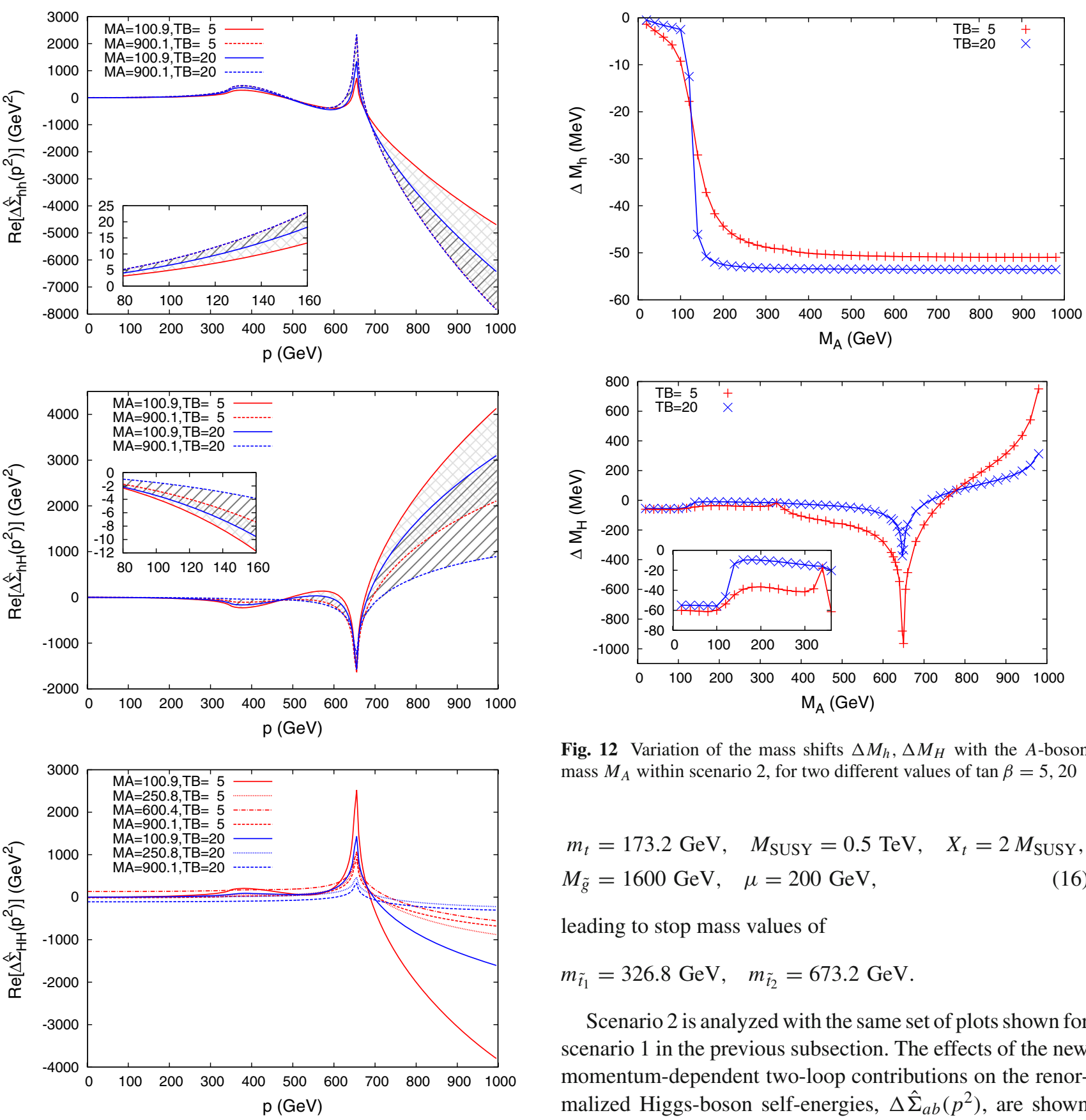

Fig. 12 Variation of the mass shifts $\Delta M_{h}, \Delta M_{H}$ with the $A$-boson mass $M_{A}$ within scenario 2, for two different values of $\tan \beta=5,20$

$$
\begin{array}{rlr}
m_{t} & =173.2 \mathrm{GeV}, \quad M_{\text {SUSY }}=0.5 \mathrm{TeV}, \quad X_{t}=2 M_{\text {SUSY }}, \\
M_{\tilde{g}} & =1600 \mathrm{GeV}, \quad \mu=200 \mathrm{GeV},
\end{array}
$$

leading to stop mass values of

$m_{\tilde{t}_{1}}=326.8 \mathrm{GeV}, \quad m_{\tilde{t}_{2}}=673.2 \mathrm{GeV}$.

Scenario 2 is analyzed with the same set of plots shown for scenario 1 in the previous subsection. The effects of the new momentum-dependent two-loop contributions on the renormalized Higgs-boson self-energies, $\Delta \hat{\Sigma}_{a b}\left(p^{2}\right)$, are shown in Fig. 10. As before, we show the results separately for the real and imaginary parts of the self-energies. An additional threshold beyond the top-mass threshold appears at $p=2 m_{\tilde{t}_{1}}$, where the discontinuity stems from the derivative of the imaginary part of the $B_{0}$ function(s). Analogously to scenario 1 , the largest contributions in the region below $200 \mathrm{GeV}$ arise in the real part of $\Delta \hat{\Sigma}_{h h}$ amounting to about $15 \mathrm{GeV}^{2}$ at $p=125 \mathrm{GeV}$, where the dependence on the value of $\tan \beta$ is rather weak.

Footnote 2 continued

$M_{1}=340 \mathrm{GeV}, M_{2}=\mu=400 \mathrm{GeV}$ leads to a SUSY spectrum that is very difficult to test at the LHC. 

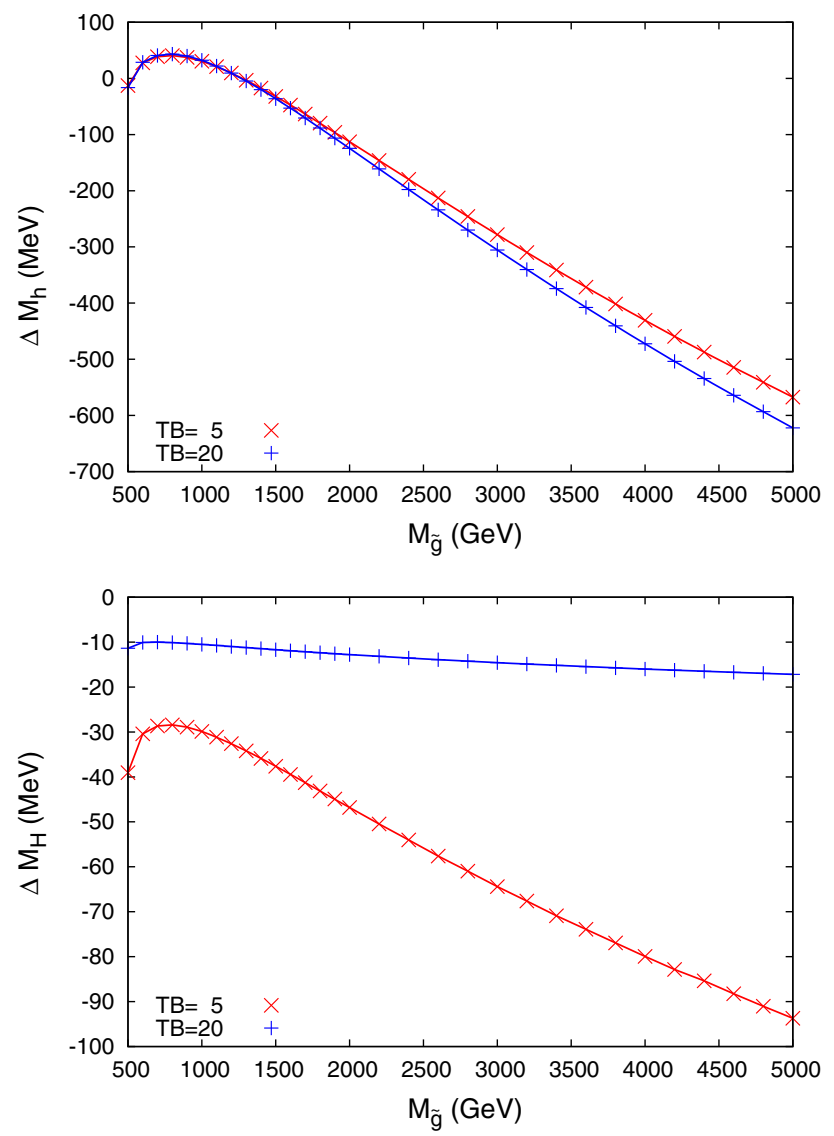

Fig. 13 Variation of the mass shifts $\Delta M_{h}, \Delta M_{H}$ with the gluino mass, within scenario 2 , for two different values of $\tan \beta=5,20$ and $M_{A}=$ $250 \mathrm{GeV}$

The dependence of $\Delta \hat{\Sigma}_{a b}\left(p^{2}\right)$ on $M_{A}$ is shown in Fig. 11, using the same line styles as in Fig. 6. The curves show the same qualitative behavior as in Fig. 10, exhibiting again the new threshold at $p=2 m_{\tilde{t}_{1}}$. In general, outside the threshold region the effects in scenario 2 are slightly smaller than in scenario 1.

We now turn to the effects on the physical neutral $\mathcal{C P}$-even Higgs boson masses. In Fig. 12 we show the results for $\Delta M_{h}$ (upper plot) and $\Delta M_{H}$ (lower plot) as a function of $M_{A}$ (with the same line styles as in Fig. 7). As can be expected from the previous figures, the effects on $M_{h}$ and $M_{H}$ are in general slightly smaller in scenario 2 than in scenario 1 , where $\Delta M_{h}$ still reaches the anticipated ILC accuracy; see Eq. (14). For $\Delta M_{H}$ around the threshold $p=2 m_{\tilde{t}_{1}}$ the largest shift of $\sim-1 \mathrm{GeV}$ can be observed. However, this shift is still below the anticipated mass resolution at the LHC [98].

Finally we analyze the dependence on $M_{\tilde{g}}$ in Fig. 13. In the upper plot we show $\Delta M_{h}$ for $\tan \beta=5$ and $\tan \beta=20$, where both values yield very similar results. As in scenario 1, "accidentally" small values of $\Delta M_{h}$ are found around $M_{\tilde{g}} \sim 1600 \mathrm{GeV}$. For larger gluino mass values the shifts induced by the new momentum-dependent two-loop corrections exceed $-500 \mathrm{MeV}$ and are thus larger than the current experimental uncertainty. The results for $\Delta M_{H}$ are shown in the lower plot. While they are roughly twice as large as in scenario 1 , they do not exceed $-100 \mathrm{MeV}$.

\section{Conclusions}

We have presented results for the leading momentumdependent $\mathcal{O}\left(\alpha_{t} \alpha_{s}\right)$ contributions to the masses of neutral $\mathcal{C P}$-even Higgs bosons in the MSSM. They are obtained by calculating the corresponding contributions to the dressed Higgs-boson propagators obtained in the Feynman-diagrammatic approach using a mixed on-shell/ $\overline{\mathrm{DR}}$ renormalization scheme. In the Higgs sector a two-loop renormalization has to be carried out for the mass of the neutral Higgs bosons and the tadpole contributions. Furthermore, renormalization of the top/stop sector at $\mathcal{O}\left(\alpha_{s}\right)$ is needed entering at the two-loop level via one-loop subrenormalization. The diagrams were generated with FeynArts and reduced to a set of basic integrals with the help of FormCalc and TwoCalc. The two-loop integrals which are analytically unknown have been calculated numerically with the program SecDec.

We have analyzed numerically the effect of the new momentum-dependent two-loop corrections on the predictions for the $\mathcal{C P}$-even Higgs boson masses. This is particularly important for the interpretation of the scalar boson discovered at the $\mathrm{LHC}$ as the light $\mathcal{C P}$-even Higgs state of the MSSM. While currently a precision below the level of $\sim 500 \mathrm{MeV}$ is reached, a reduction by about an order of magnitude can be expected at the future $e^{+} e^{-}$International Linear Collider (ILC).

In our numerical analysis we found that the effects on the light $\mathcal{C P}$-even Higgs boson mass, $M_{h}$, depend strongly on the value of the gluino mass, $M_{\tilde{g}}$. For values of $M_{\tilde{g}} \sim 1.5 \mathrm{TeV}$ corrections to $M_{h}$ of about $-50 \mathrm{MeV}$ are found, at the level of the anticipated future ILC accuracy. For very large gluino masses, $M_{\tilde{g}} \gtrsim 4 \mathrm{TeV}$, on the other hand, substantially larger corrections are found, at the level of the current experimental accuracy. Consequently, this type of momentum-dependent two-loop corrections should be taken into account in precision analyses interpreting the discovered Higgs boson in the MSSM.

For the heavy $\mathcal{C} \mathcal{P}$-even Higgs boson mass, $M_{H}$, the effects are mostly below current and future anticipated accuracies. Only close to thresholds, e.g. around $p=2 m_{\tilde{t}_{1}}$, larger corrections to $M_{H}$ around $\sim 1 \mathrm{GeV}$ are found.

The new results of $\mathcal{O}\left(\alpha_{t} \alpha_{S}\right)$ have been implemented into the program FeynHiggs. A detailed description of our calculation will be presented in a forthcoming publication [99].

Acknowledgments We thank S. Paßehr for help with the interfaces to TwoCalc and FormCalc, S. di Vita for numerical comparisons and G. Weiglein for helpful discussions. The work of S.H. was supported by 
the Spanish MICINN's Consolider-Ingenio 2010 Program under Grant MultiDark No. CSD2009-00064.

Open Access This article is distributed under the terms of the Creative Commons Attribution License which permits any use, distribution, and reproduction in any medium, provided the original author(s) and the source are credited.

Funded by $\mathrm{SCOAP}^{3}$ / License Version CC BY 4.0.

\section{Appendix: Renormalization and counterterms}

Renormalization and calculation of the renormalized selfenergies is performed in the $\left(\phi_{1}, \phi_{2}\right)$ basis, which has the advantage that the mixing angle $\alpha$ does not appear and expressions are in general simpler.

Field renormalization is performed by assigning one renormalization constant for each doublet,

$\mathcal{H}_{1} \rightarrow\left(1+\frac{1}{2} \delta Z_{\mathcal{H}_{1}}\right) \mathcal{H}_{1}, \quad \mathcal{H}_{2} \rightarrow\left(1+\frac{1}{2} \delta Z_{\mathcal{H}_{2}}\right) \mathcal{H}_{2}$,

which can be expanded to one- and two-loop order according to

$\delta Z_{\mathcal{H}_{1}}=\delta Z_{\mathcal{H}_{1}}^{(1)}+\delta Z_{\mathcal{H}_{1}}^{(2)}, \quad \delta Z_{\mathcal{H}_{2}}=\delta Z_{\mathcal{H}_{2}}^{(1)}+\delta Z_{\mathcal{H}_{2}}^{(2)}$

The field renormalization constants appearing in (4) are then given by

$\delta Z_{\phi_{1}}^{(i)}=\delta Z_{\mathcal{H}_{1}}^{(i)}, \quad \delta Z_{\phi_{2}}^{(i)}=\delta Z_{\mathcal{H}_{2}}^{(i)}$,

$\delta Z_{\phi_{1} \phi_{2}}^{(i)}=\frac{1}{2}\left(\delta Z_{\mathcal{H}_{1}}^{(i)}+\delta Z_{\mathcal{H}_{2}}^{(i)}\right)$.

The mass counterterms $\delta m_{a b}^{2(i)}$ in (4) are derived from the Higgs potential, including the tadpoles, by the following parameter renormalization:

$M_{A}^{2} \rightarrow M_{A}^{2}+\delta M_{A}^{2(1)}+\delta M_{A}^{2(2)}$,

$T_{1} \rightarrow T_{1}+\delta T_{1}^{(1)}+\delta T_{1}^{(2)}$,

$M_{Z}^{2} \rightarrow M_{Z}^{2}+\delta M_{Z}^{2(1)}+\delta M_{Z}^{2(2)}$

$T_{2} \rightarrow T_{2}+\delta T_{2}^{(1)}+\delta T_{2}^{(2)}$

$\tan \beta \rightarrow \tan \beta\left(1+\delta \tan \beta^{(1)}+\delta \tan \beta^{(2)}\right)$.

The parameters $T_{1}$ and $T_{2}$ are the terms linear in $\phi_{1}$ and $\phi_{2}$ in the Higgs potential. The renormalization of the $Z$ mass $M_{Z}$ does not contribute to the $\mathcal{O}\left(\alpha_{s} \alpha_{t}\right)$ corrections we are pursuing here; it is listed, however, for completeness.

The basic renormalization constants for parameters and fields have to be fixed by renormalization conditions according to a renormalization scheme. Here we choose the on-shell scheme for the parameters and the $\overline{\mathrm{DR}}$ scheme for field renormalization and give the expressions for the two-loop part.

The tadpole coefficients are chosen to vanish at all orders; hence their two-loop counterterms follow from

$T_{1,2}^{(2)}+\delta T_{1,2}^{(2)}=0, \quad$ i.e. $\quad \delta T_{1}^{(2)}=-T_{1}^{(2)}, \quad \delta T_{2}^{(2)}=-T_{2}^{(2)}$, where $T_{1}^{(2)}, T_{2}^{(2)}$ are obtained from the two-loop tapole diagrams. The two-loop renormalization constant of the $A$ boson mass reads

$\delta M_{A}^{2(2)}=\operatorname{Re} \Sigma_{A A}^{(2)}\left(M_{A}^{2}\right)$

in terms of the $A$-boson unrenormalized self-energy $\Sigma_{A A}$. The appearance of a non-zero momentum in the self-energy goes beyond the $\mathcal{O}\left(\alpha_{t} \alpha_{s}\right)$ corrections evaluated in Refs. [36$38,44]$.

For the renormalization constants $\delta Z_{\mathcal{H}_{1}}, \delta Z_{\mathcal{H}_{2}}$ and $\delta \tan \beta$ several choices are possible; see the discussion in [100-102]. As shown there, the most convenient choice is a $\overline{\mathrm{DR}}$ renormalization of $\delta \tan \beta, \delta Z_{\mathcal{H}_{1}}$ and $\delta Z_{\mathcal{H}_{2}}$, which reads at the two-loop level

$$
\begin{gathered}
\delta Z_{\mathcal{H}_{1}}^{(2)}=\delta Z_{\mathcal{H}_{1}}^{(2) \overline{\mathrm{DR}}}=-\left[\operatorname{Re} \Sigma_{\phi_{1}}^{\prime(2)}\right]_{\mid p^{2}=0}^{\mathrm{div}}, \\
\delta Z_{\mathcal{H}_{2}}^{(2)}=\delta Z_{\mathcal{H}_{2}}^{(2) \overline{\mathrm{DR}}}=-\left[\operatorname{Re} \Sigma_{\phi_{2}}^{\prime(2)}\right]_{\mid p^{2}=0}^{\mathrm{div}}, \\
\delta \tan \beta^{(2)}=\delta \tan \beta^{(2) \overline{\mathrm{DR}}}=\frac{1}{2}\left(\delta Z_{\mathcal{H}_{2}}^{(2)}-\delta Z_{\mathcal{H}_{1}}^{(2)}\right) .
\end{gathered}
$$

The term in Eq. (23c) is in general not the proper expression beyond one-loop order even in the $\overline{\mathrm{DR}}$ scheme. For our approximation, however, with only the top Yukawa coupling at the two-loop level, it is the correct $\overline{\mathrm{DR}}$ form $[103,104]$.

The two-loop mass counterterms in the self-energies (4) are now expressed in terms of the parameter renormalization constants determined above as follows:

$$
\begin{aligned}
\delta m_{\phi_{1}}^{2(2)}= & \delta M_{Z}^{2(2)} \cos ^{2} \beta+\delta M_{A}^{2(2)} \sin ^{2} \beta \\
& -\delta T_{1}^{(2)} \frac{e}{2 M_{W} s_{\mathrm{W}}} \cos \beta\left(1+\sin ^{2} \beta\right) \\
& +\delta T_{2}^{(2)} \frac{e}{2 M_{W} s_{\mathrm{W}}} \cos ^{2} \beta \sin \beta \\
& +2 \delta \tan \beta^{(2)} \cos ^{2} \beta \sin ^{2} \beta\left(M_{A}^{2}-M_{Z}^{2}\right) \\
\delta m_{\phi_{1} \phi_{2}}^{2(2)}= & -\left(\delta M_{Z}^{2(2)}+\delta M_{A}^{2(2)}\right) \sin \beta \cos \beta \\
& -\delta T_{1}^{(2)} \frac{e}{2 M_{W} s_{\mathrm{W}}} \sin ^{3} \beta-\delta T_{2}^{(2)} \frac{e}{2 M_{W} s_{\mathrm{W}}} \cos ^{3} \beta \\
& -\delta \tan \beta^{(2)} \cos \beta \sin ^{3} \cos 2 \beta\left(M_{A}^{2}+M_{Z}^{2}\right) \\
\delta m_{\phi_{2}}^{2(2)}= & \delta M_{Z}^{2(2)} \sin ^{2} \beta+\delta M_{A}^{2(2)} \cos { }^{2} \beta \\
& +\delta T_{1}^{(2)} \frac{e}{2 M_{W} s_{\mathrm{W}}} \sin ^{2} \beta \cos \beta \\
& -\delta T_{2}^{(2)} \frac{e}{2 M_{W} s_{\mathrm{W}}} \sin ^{2} \beta\left(1+\cos ^{2} \beta\right) \\
& -2 \delta \tan \beta^{(2)} \cos ^{2} \beta \sin ^{2} \beta\left(M_{A}^{2}-M_{Z}^{2}\right)
\end{aligned}
$$

Note that the $Z$-mass counterterm is kept for completeness; it does not contribute in the approximation of $\mathcal{O}\left(\alpha_{s} \alpha_{t}\right)$ considered here. 


\section{References}

1. G. Aad et al. [ATLAS Collaboration], Phys. Lett. B 716, 1 (2012). arXiv:1207.7214 [hep-ex]

2. S. Chatrchyan et al. [CMS Collaboration], Phys. Lett. B 716, 30 (2012). arXiv:1207.7235 [hep-ex]

3. P. Bargassa, Talk given at "Rencontres de Moriond EW 2014". https://indico.in2p3.fr/getFile.py/access?contribId=189\& sessionId=0\&resId $=1 \&$ materialId $=$ slides $\&$ confId $=9116$

4. M. Flowerdew, Talk given at "Rencontres de Moriond EW 2014". https://indico.in2p3.fr/getFile.py/access?contribId=169\& sessionId $=0 \&$ res $I d=0 \&$ materialId $=$ slides $\&$ conf $I d=9116$

5. P. Thompson, Talk given at "Rencontres de Moriond EW 2014". https://indico.in2p3.fr/getFile.py/access?contribId=220\& sessionId $=8 \&$ resId $=0 \&$ materialId $=$ slides $\& \operatorname{confId}=9116$

6. K. Einsweiler, Talk given at "Rencontres de Moriond EW 2014". https://indico.in2p3.fr/getFile.py/access?contribId=227\& sessionId=1\&resId=1\&materialId=slides\& confId=9116

7. H. Nilles, Phys. Rep. 110, 1 (1984)

8. H. Haber, G. Kane, Phys. Rep. 117, 75 (1985)

9. R. Barbieri, Riv. Nuovo Cim. 11, 1 (1988)

10. A. Djouadi, Phys. Rep. 459, 1 (2008). arXiv:hep-ph/0503173

11. S. Heinemeyer, Int. J. Mod. Phys. A 21, 2659 (2006). arXiv:hep-ph/0407244

12. S. Heinemeyer, W. Hollik, G. Weiglein, Phys. Rep. 425, 265 (2006). arXiv:hep-ph/0412214

13. G. Degrassi, S. Heinemeyer, W. Hollik, P. Slavich, G. Weiglein, Eur. Phys. J. C 28, 133 (2003). arXiv:hep-ph/0212020

14. T. Hahn, S. Heinemeyer, W. Hollik, H. Rzehak, G. Weiglein, Phys. Rev. Lett. 112, 141801 (2014). arXiv:1312.4937 [hep-ph]

15. O. Buchmueller et al., Eur. Phys. J. C 74, 2809 (2014). arXiv: 1312.5233 [hep-ph]

16. [LEP Higgs Working Group], Phys. Lett. B 565, 61 (2003). arXiv:hep-ex/0306033

17. [LEP Higgs Working Group], Eur. Phys. J. C 47, 547 (2006). arXiv:hep-ex/0602042

18. [CMS Collaboration], CMS-PAS-HIG-13-021

19. D. Asner et al., ILC Higgs Snowmass White Paper, CNUM: C1307-29.2. arXiv: 1310.0763 [hep-ph]

20. H. Baer et al., The International Linear Collider Technical Design Report Volume 2: Physics. arXiv:1306.6352 [hep-ph]

21. [LHC/ILC Study Group], G. Weiglein et al., Phys. Rep. 426, 47 (2006). arXiv:hep-ph/0410364

22. A. De Roeck et al., Eur. Phys. J. C 66, 525 (2010). arXiv:0909.3240 [hep-ph]

23. K. Desch, E. Gross, S. Heinemeyer, G. Weiglein, L. Zivkovic, JHEP 0409, 062 (2004). arXiv:hep-ph/0406322

24. M. Frank, T. Hahn, S. Heinemeyer, W. Hollik, H. Rzehak, G. Weiglein, JHEP 0702, 047 (2007). arXiv:hep-ph/0611326

25. S. Heinemeyer, W. Hollik, H. Rzehak, G. Weiglein, Phys. Lett. B 652, 300 (2007). arXiv:0705.0746 [hep-ph]

26. D. Demir, Phys. Rev. D 60, 055006 (1999). arXiv:hep-ph/9901389

27. A. Pilaftsis, C. Wagner, Nucl. Phys. B 553, 3 (1999). arXiv:hep-ph/9902371

28. J. Ellis, G. Ridolfi, F. Zwirner, Phys. Lett. B 257, 83 (1991)

29. Y. Okada, M. Yamaguchi, T. Yanagida, Prog. Theor. Phys. 85, 1 (1991)

30. H. Haber, R. Hempfling, Phys. Rev. Lett. 66, 1815 (1991)

31. A. Brignole, Phys. Lett. B 281, 284 (1992)

32. P. Chankowski, S. Pokorski, J. Rosiek, Phys. Lett. B 286, 307 (1992)

33. P. Chankowski, S. Pokorski, J. Rosiek, Nucl. Phys. B 423, 437 (1994). arXiv:hep-ph/9303309

34. A. Dabelstein, Nucl. Phys. B 456, 25 (1995). arXiv:hep-ph/9503443
35. A. Dabelstein, Z. Phys. C 67, 495 (1995). arXiv:hep-ph/9409375

36. S. Heinemeyer, W. Hollik, G. Weiglein, Phys. Rev. D 58, 091701 (1998). arXiv:hep-ph/9803277

37. S. Heinemeyer, W. Hollik, G. Weiglein, Phys. Lett. B 440, 296 (1998). arXiv:hep-ph/9807423

38. S. Heinemeyer, W. Hollik, G. Weiglein, Eur. Phys. J. C 9, 343 (1999). arXiv:hep-ph/9812472

39. S. Heinemeyer, W. Hollik, G. Weiglein, Phys. Lett. B 455, 179 (1999). arXiv:hep-ph/9903404

40. S. Heinemeyer, W. Hollik, H. Rzehak, G. Weiglein, Eur. Phys. J. C 39, 465 (2005). arXiv:hep-ph/0411114

41. M. Carena, H. Haber, S. Heinemeyer, W. Hollik, C. Wagner, G. Weiglein, Nucl. Phys. B 580, 29 (2000). arXiv:hep-ph/0001002

42. R. Zhang, Phys. Lett. B 447, 89 (1999). arXiv:hep-ph/9808299

43. J. Espinosa, R. Zhang, JHEP 0003, 026 (2000). arXiv:hep-ph/9912236

44. G. Degrassi, P. Slavich, F. Zwirner, Nucl. Phys. B 611, 403 (2001). arXiv:hep-ph/0105096

45. R. Hempfling, A. Hoang, Phys. Lett. B 331, 99 (1994). arXiv:hep-ph/9401219

46. A. Brignole, G. Degrassi, P. Slavich, F. Zwirner, Nucl. Phys. B 631, 195 (2002). arXiv:hep-ph/0112177

47. J. Espinosa, R. Zhang, Nucl. Phys. B 586, 3 (2000). arXiv:hep-ph/0003246

48. J. Espinosa, I. Navarro, Nucl. Phys. B 615, 82 (2001). arXiv:hep-ph/0104047

49. A. Brignole, G. Degrassi, P. Slavich, F. Zwirner, Nucl. Phys. B 643, 79 (2002). arXiv:hep-ph/0206101

50. G. Degrassi, A. Dedes, P. Slavich, Nucl. Phys. B 672, 144 (2003). arXiv:hep-ph/0305127

51. M. Carena, J. Espinosa, M. Quirós, C. Wagner, Phys. Lett. B 355, 209 (1995). arXiv:hep-ph/9504316

52. M. Carena, M. Quirós, C. Wagner, Nucl. Phys. B 461, 407 (1996). arXiv:hep-ph/9508343

53. J. Casas, J. Espinosa, M. Quirós, A. Riotto, Nucl. Phys. B 436, 3 (1995). arXiv:hep-ph/9407389 [Erratum-ibid. B 439, 466 (1995)]

54. S. Martin, Phys. Rev. D 71, 016012 (2005). arXiv:hep-ph/0405022

55. S. Martin, Phys. Rev. D 65, 116003 (2002). arXiv:hep-ph/0111209

56. S. Martin, Phys. Rev. D 66, 096001 (2002). arXiv:hep-ph/0206136

57. S. Martin, Phys. Rev. D 67, $095012 \quad$ (2003). arXiv:hep-ph/0211366

58. S. Martin, Phys. Rev. D 68, $075002 \quad$ (2003). arXiv:hep-ph/0307101

59. S. Martin, Phys. Rev. D 70, 016005 (2004). arXiv:hep-ph/0312092

60. S. Martin, Phys. Rev. D 71, 116004 (2005). arXiv:hep-ph/0502168

61. S. Martin, Phys. Rev. D 75, 055005 (2007). arXiv:hep-ph/0701051

62. S. Martin, D. Robertson, Comput. Phys. Commun. 174, 133 (2006). arXiv:hep-ph/0501132

63. R. Harlander, P. Kant, L. Mihaila, M. Steinhauser, Phys. Rev. Lett. 100, 191602 (2008)

64. R. Harlander, P. Kant, L. Mihaila, M. Steinhauser, Phys. Rev. Lett. 101, 039901 (2008). arXiv:0803.0672 [hep-ph]

65. R. Harlander, P. Kant, L. Mihaila, M. Steinhauser, JHEP 1008, 104 (2010). arXiv:1005.5709 [hep-ph]

66. S. Heinemeyer, W. Hollik, G. Weiglein, Comput. Phys. Commun. 124, 76 (2000). arXiv:hep-ph/9812320

67. T. Hahn, S. Heinemeyer, W. Hollik, H. Rzehak, G. Weiglein, Comput. Phys. Commun. 180, 1426 (2009). See http://www.feynhiggs. de 
68. J. Küblbeck, M. Böhm, A. Denner, Comput. Phys. Commun. 60, 165 (1990)

69. T. Hahn, Comput. Phys. Commun. 140, 418 (2001). arXiv:hep-ph/0012260

70. T. Hahn, C. Schappacher, Comput. Phys. Commun. 143, 54 (2002). arXiv:hep-ph/0105349

71. The program and the user's guide are available via http://www. feynarts.de

72. T. Fritzsche, T. Hahn, S. Heinemeyer, F. von der Pahlen, H. Rzehak, C. Schappacher, Comput. Phys. Commun. 185, 1529 (2014). arXiv:1309.1692 [hep-ph]

73. T. Hahn, M. Pérez-Victoria, Comput. Phys. Commun. 118, 153 (1999). arXiv:hep-ph/9807565

74. G. Weiglein, R. Scharf, M. Böhm, Nucl. Phys. B 416, 606 (1994). arXiv:hep-ph/9310358

75. G. Weiglein, R. Mertig, R. Scharf, M. Böhm, in New Computing Techniques in Physics Research, vol. 2, ed. D. Perret-Gallix (World Scientific, Singapore, 1992), p. 617

76. G. 't Hooft, M. Veltman, Nucl. Phys. B 153, 365 (1979)

77. A.I. Davydychev, J.B. Tausk, Nucl. Phys. B 397, 123 (1993)

78. J. Carter, G. Heinrich, Comput. Phys. Commun. 182, 1566 (2011). arXiv:1011.5493 [hep-ph]

79. S. Borowka, J. Carter, G. Heinrich, Comput. Phys. Commun. 184, 396 (2013). arXiv:1204.4152 [hep-ph]

80. W. Hollik, H. Rzehak, Eur. Phys. J. C 32, 127 (2003). arXiv:hep-ph/0305328

81. S. Heinemeyer, H. Rzehak, C. Schappacher, Phys. Rev. D 82, 075010 (2010). arXiv:1007.0689 [hep-ph]

82. T. Fritzsche, S. Heinemeyer, H. Rzehak, C. Schappacher, Phys. Rev. D 86, 035014 (2012). arXiv:1111.7289 [hep-ph]

83. D.J. Broadhurst, Z. Phys, C 47, 115 (1990)

84. A.I. Davydychev, V.A. Smirnov, J.B. Tausk, Nucl. Phys. B 410, 325 (1993). arXiv:hep-ph/9307371

85. R. Scharf, J.B. Tausk, Nucl. Phys. B 412, 523 (1994)
86. F.A. Berends, J.B. Tausk, Nucl. Phys. B 421, 456 (1994)

87. S. Bauberger, Diploma thesis (1994)

88. S. Bauberger, M. Böhm, Nucl. Phys. B 445, 25 (1995). arXiv:hep-ph/9501201

89. S. Laporta, E. Remiddi, Nucl. Phys. B 704, 349 (2005). arXiv:hep-ph/0406160

90. E. Remiddi, L. Tancredi, Nucl. Phys. B 880, 343 (2014). arXiv:1311.3342 [hep-ph]

91. The code is available at http://secdec.hepforge.org/

92. T. Binoth, G. Heinrich, Nucl. Phys. B 585, 741 (2000). arXiv:hep-ph/0004013

93. G. Heinrich, Int. J. Mod. Phys. A 23, 1457 (2008). arXiv:0803.4177 [hep-ph]

94. T. Hahn, Comput. Phys. Commun. 168, 78 (2005). arXiv:hep-ph/0404043

95. S. Borowka, G. Heinrich, Comput. Phys. Commun. 184, 2552 (2013). arXiv:1303.1157 [hep-ph]

96. M. Carena, S. Heinemeyer, O. Stål, C. Wagner, G. Weiglein, Eur. Phys. J. C 73, 2552 (2013). arXiv:1302.7033 [hep-ph]

97. M. Carena, S. Heinemeyer, C. Wagner, G. Weiglein, Eur. Phys. J. C 26, 601 (2003). arXiv:hep-ph/0202167

98. S. Gennai et al., Eur. Phys. J. C 52, 383 (2007). arXiv:0704.0619 [hep-ph]

99. S. Borowka, G. Heinrich, W. Hollik, in preparation

100. M. Frank, S. Heinemeyer, W. Hollik, G. Weiglein, arXiv:hep-ph/0202166

101. A. Freitas, D. Stöckinger, Phys. Rev. D 66, 095014 (2002). arXiv:hep-ph/0205281

102. K. Ender, T. Graf, M. Muhlleitner, H. Rzehak, Phys. Rev. D 85, 075024 (2012). arXiv:1111.4952 [hep-ph]

103. M. Sperling, D. Stöckinger, A. Voigt, JHEP 1307, 132 (2013). arXiv:1305.1548 [hep-ph]

104. M. Sperling, D. Stöckinger, A. Voigt, JHEP 1401, 068 (2014). arXiv:1310.7629 [hep-ph] 